\title{
IOT based Smart Notice Board
}

\author{
Gaurav Bhardwaj \\ UG Student, Department of ECE \\ MIT School of Engineering \\ Pune, Maharashtra, India
}

\author{
Gunjan Sahu \\ UG Student, Department of ECE \\ MIT School of Engineering \\ Pune, Maharashtra, India
}

\author{
Rajan Kumar Mishra \\ UG Student, Department of ECE \\ MIT School of Engineering \\ Pune, Maharashtra, India
}

\begin{abstract}
IOT is the network of physical "things" or object that contain embedded technology to interface and sense to move with their internal states or the external setting. Automation is the most often spelled term within the field of electronics. The hunger for automation brought several revolutions within the existing technologies. Notice board could be a primary factor in any establishment or public places like bus stations, railway stations, colleges, malls etc. Sticking out numerous notices day to day could be a tough method. A separate person is needed to take care of this notice display. This project is regarding advanced wireless notice board. In IOT based Web Controlled Notice Board, Internet is employed to wirelessly send the message from Browser to the display. A local web server is created, this could be a global server over net. At the PIC microcontroller, LED matrix is used to display message and flask for receiving the message over network. Whenever microcontroller receives any wireless message from GSM module, it displays on the LED matrix. The Internet of Things (IOT) belief system can be looked as an exceptionally unique and radically distributed networked system composed of a very large number of identifiable smart objects. These objects can convey and to interface among themselves, with end- users or different elements in the system. Entering the era of Internet of Things, the use of small, shoddy and flexible computer hardware that allow end-user programming become present. One of them, considered in this, is the PIC microcontroller, fully customizable and programmable small computer board. Relative investigation of its key components and exhibitions with some of current existing IOT prototype platforms have shown that despite few disadvantages, the PIC microcontroller remains an modest with its effectively utilization in diverse range of research applications in IOT vision.
\end{abstract}

Keywords-LED Matrix; PIC Microcontroller; SPI; GSM Modem.

\section{INTRODUCTION}

The main purposes to design this electronic notice board system is to interface it with user's mobile phones for displaying the latest information. In other words, the user can easily send the message or information for displaying from remote areas. In this system, the user sends the information or data from remote areas and this information or data is received by the sim loaded into GSM modem which is basically receiving end. This system is designed with PIC16F microcontroller, which is interfaced with GSM modem and level shifter through serial cable. LED Matrix is also used in this system for displaying the information or data.

Max 232 is a 16-pin dip package, which consists of three major blocks and is powered by 5 volt's supply. It is mostly used in embedded system for serial communication. The problem is occurred when we communicate between TTL logic and CMOS logic because, here in wireless electronic notice board we are communicating between GSM modem and microcontroller therefore the problem would be occurred. For solving this problem, the max 232 is used in this specific wireless electronic notice board.

The GSM modem is a wireless modem, which can't work without wireless network. This modem works like a dialup modem and sim is required for communication. In dialup modem the data is send or received through the fix telephonic line but in GSM modem data is send or received though the radio waves

\section{METHODOLOGY}

The main function of the proposed system is to develop a Digital notice board that display message sent from the user through mobile phone (SMS) and to design a simple, user friendly system, which can receive and display message/information in a particular manner with respect to date and time which will help the user to easily keep the track of notice board every day and each time he uses the system. System consist of two section called as sender and receiver. Sender is responsible for sending valuable information through the GSM protocol.

In order to access Digital notice board the sender must enter into the corresponding mobile number [3]. When the user enter correct mobile number, the message can be typed and get space for the information transmission.

To make the proposed system more user friendly we make an android application .By using this application sender can directly enter the message.

In receiver section, PIC microcontroller is connected on GSM module for accessing the sim card.

It is a capable little device that enables people of all ages to explore radio waves transmission and reception.

We use here embedded C language and GSM protocol as the basic working principle.

\section{SYSTEM REQUIREMENTS}

\section{A. Transformer:}

In this wireless electronic notice board the transformer, which consists of two winding's primary and secondary is used for converting the $220 \mathrm{~V}$ to $24 \mathrm{~V}$ ac because this system is directly connected to the power supply.

\section{B. Voltage Regulator:}

The voltage regulator is used for providing the fix 12 volts, DC to the microcontroller and LCD display. In the absence of voltage regulator, the higher voltage may be damage the LED Matrix display or microcontroller and in this system these two components are too much important. 


\section{MAX232:}

The problem is occurred when we communicate between TTL logic and CMOS logic because, here in wireless electronic notice board we are communicating between GSM modem and microcontroller therefore the problem would be occurred. For solving this problem, the max 232 is used in this specific wireless electronic notice board.

\section{GSM Modem:}

GSM modem is a wireless modem as shown in Fig. 1. It works on wireless network. This modem works like a dialup modem and sim is required for communication. In dialup modem the data is send or received through the fix telephonic line but in GSM modem data is send or received though the radio waves.

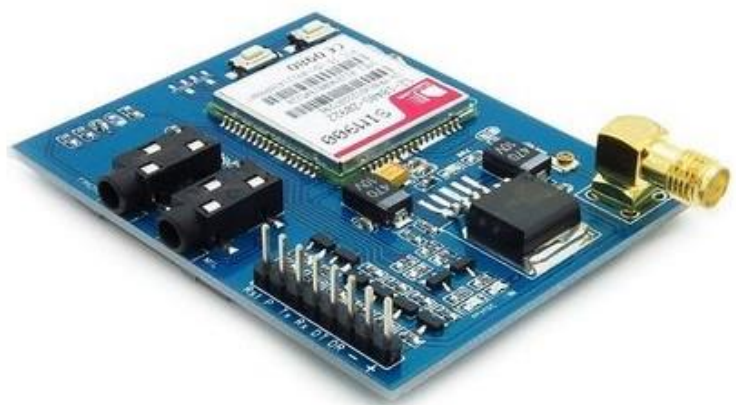

Fig. 1: SIM900A GSM Module [8]

\section{E. LED Matrix Display:}

In this system we have used a led matrix of $8 \times 8$ resolution. $8 \times 8$ matrix consists of 64 dots or pixels. There is a LED for each pixel and these LEDs are connected to total of 16 pins. The whole LED matrix operates on 12 volt's DC. Every LED is connected through a resistor to limit the current through LEDs, also a current driver circuit can be used to ensure a uniform brightness of LEDs

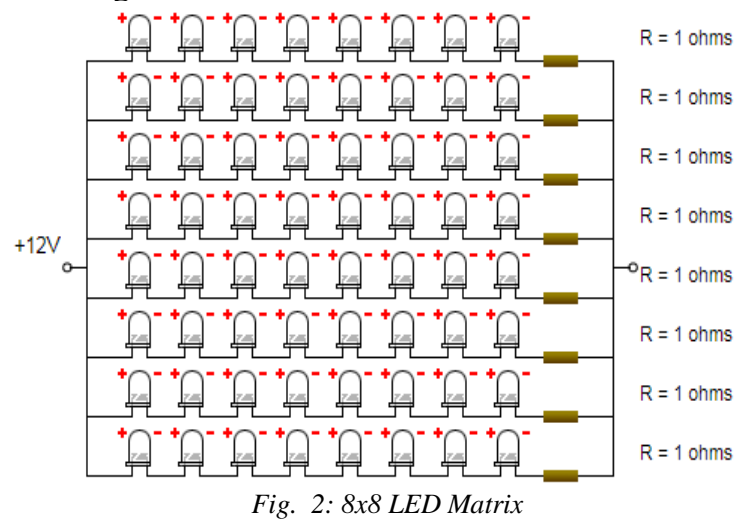

IV. MICROCONTROLLER

This system is based on PIC16F877A microcontroller developed by Microchip. It is 8-bit microcontroller which has selfIt is 8-bit microcontroller which has self-programming, 256 bytes of EEPROM and $40 \mathrm{MHz}$ of maximum speed. It also has 8 channel of analog to digital converter (A/D), two PWM functions and synchronous serial port for serial communication. The synchronous serial port can also be configured either for 3 wire serials peripheral interface (SPI) or 2 wire inter integrated circuit bus and addressable universal asynchronous transmitter receiver.
PIC16F877A uses FLASH memory technology so that it can be write-erase until thousand times. The superiority of this RISC Microcontroller as compared to other microcontroller is its 8-bit operation especially at a speed of $40 \mathrm{MHz}$ and its code compression.

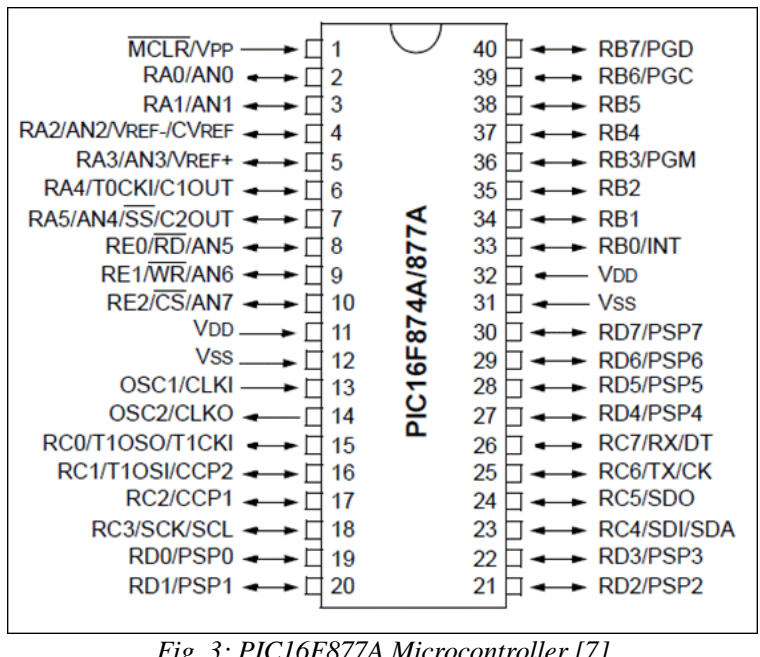

\section{A. Programming PIC Microcontroller:}

PIC microcontroller can be programmed with different software's that are available in the market. There are people who still use Assembly language to program PIC MCUs. However most of the programmers use embedded $C$ language to program the microcontroller, because of it's easy to read nature and code optimization using functions and header files. In order to program the PIC microcontroller we will need an IDE where the programming takes place. An IDE has a compiler, where our program gets converted into binary format. Using the boot loader protocol HEX file (executable file) is uploaded in the flash memory of microcontroller.

\section{SERIAL COMMUNICATION}

GSM protocol works on serial communication protocol. Serial communication is performed in PIC microcontroller with the SPI (Serial Peripheral Interface) protocol. It is considered as one of the fastest serial data transfer interfaces for the embedded system.

\section{A. Serial Peripheral Interface (SPI):}

SPI is a synchronous protocol based on the master-slave communication type. The master device (usually a microcontroller) controls slave devices. Because the slave device takes instructions from the master device. It allows transmission of data from a master device to one or more slave devices and from slave devices to master devices over short distances at high speeds $(\mathrm{MHz})$. It consists of 4 fours but three-wire communication is also possible.

There are four SPI modes which describe the relationship between the phase of the clock line and the phase of the MISO/MOSI lines. In order to successfully communicate using SPI, the master and slave must operate using the same mode. 


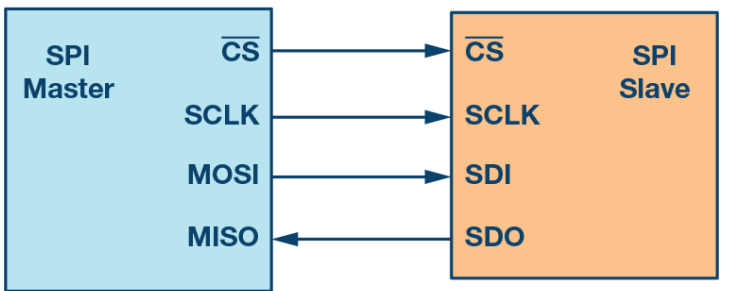

Fig. 4: SPI Protocol [7]

VI. APPLICATIONS

This system can be implemented for commercial purpose as well as for individual use. Further modification in the system can also led to reminder system, in which an event can be scheduled using the date and time and accordingly the system will alert you with appropriate information about the event. Generally it can be can be used in classrooms, schools, seminar halls to updates.

\section{THREATS TO THE SYSTEM}

Currently this system is totally dependent on the sim card service provider to perform the transmission and reception, so any problem with the service provider will affect our system. Secondly this system has a wide bandwidth, which can be optimized by modifying the protocols or platform of the system. This provides a discussion on present trends in technology and how exactly, simple carry-to-use devices play a vital role in day-to-day life.

\section{COUnTER Methods To AvoId Threats}

Modifying the same concept on different platform by using different and more efficient technology might overcome the current threats of the system. Instead of GSM module this system can be connected to cloud and make it more efficient.

\section{CONCLUSION}

The system has been successfully tested with troubleshooting to the best of our knowledge. Each block present in it has been reasoned and justified. The project is very cost efficient and marketable and the components used are very simple and easily available in the market. We believe that this system can become commercial and can be used in places such as colleges, banks railway station etc. Finally we conclude that this project being based on the widely used GSM technology has further scope for future development and research and can be modified according to its application.

\section{ACKNOWLEDGMENT}

This work is based on our project and research carried out at MIT School Of Engineering's "Microchip Lab". We thank Prof. Dr. Dhananjay Upasani for his support and guidance throughout the research.

\section{REFERENCES}

[1] Dharmendra Kumar Sharma and Vineet Tiwari, "Small and medium range wireless electronic notice board using Bluetooth and ZigBee" IEEE 2015.

[2] Neeraj Khera and Divya Shukla "Development of simple and low cost Android based wireless notice board" IEEE 2016.

[3] Aniket Pramanik, Rishikesh and Vikash Nagar "GSM based Smart home and digital notice board" IEEE 2016.

[4] Kruthika Simha, Shreya and Chethan Kumar "Electronic notice board with multiple output display" IEEE 2017

[5] S. Rubin Bose and J. Jasper Prem "Design and Implementation of Digital Notice Board Using IoT" IJRIER 2017.

[6] M. Arun, P. Monika and G. Lavanya "Raspberry Pi Controlled Smart e-Notice Board using Arduino" IJCAT 2017

[7] PIC16F87XA Datasheet By Microchip

[8] SIM900A_HD_V1.01 Datasheet By SIMCOM 